Si mul taneous proj ecti on and detecti on system of four different frequencies for mi crowave i magi ng refl ect omet ry in Large Hel i cal Devi ce

\begin{tabular}{|l|l|}
\hline $\begin{array}{l}\text { j our nal or } \\
\text { publ i cat i on t i t l e }\end{array}$ & Revi ew of Sci ent i f i c I nst r ument s \\
\hline vol une & Vol . 81 \\
\hline page r ange & 10D915- 1 - 10D915- 4 \\
\hline year & 2010 - 01- 01 \\
\hline URL & ht t p: //hdl . handl e. net /10655/6453 \\
\hline
\end{tabular}




\title{
Simultaneous projection and detection system of four different frequencies for microwave imaging reflectometry in Large Helical Device ${ }^{a)}$
}

\author{
T. Yoshinaga, ${ }^{1, b)}$ Y. Nagayama, ${ }^{1}$ D. Kuwahara,${ }^{2}$ H. Tsuchiya, ${ }^{1}$ S. Yamaguchi, ${ }^{3}$ Y. Kogi, ${ }^{4}$ \\ S. Tsuji-lio, ${ }^{2}$ and A. Mase ${ }^{5}$ \\ ${ }^{1}$ National Institute for Fusion Science, 322-6 Oroshi, Toki 509-5292, Japan \\ ${ }^{2}$ Tokyo Institute of Technology, 2-12-1 Ookayama, Meguro 152-8550, Japan \\ ${ }^{3}$ Kansai University, 3-3-35 Yamate, Suita 564-8680, Japan \\ ${ }^{4}$ Fukuoka Institute of Technology, 3-30-1 Wajiro-Higashi, Fukuoka 811- 0295, Japan \\ ${ }^{5}$ Kyushu University, 6-1 Kasuga-Koen, Kasuga 816-8680, Japan
}

(Presented 18 May 2010; received 17 May 2010; accepted 3 August 2010; published online 18 October 2010)

A simultaneous projection/detection system of four different frequencies for microwave imaging reflectometry (MIR) was developed for three-dimensional observation of electron density fluctuations in the Large Helical Device (LHD). The microwave with four frequency components at $60.410,61.808,63.008$, and $64.610 \mathrm{GHz}$ is projected in a continuous-wave mode to illuminate the target LHD plasma. A two-dimensional horn-antenna mixer array (2D HMA) receives the reflected wave from the plasma as well as the wave from the local oscillator operating at $55.800 \mathrm{GHz}$. The first intermediate frequency (IF) signals at 4.610, 6.008, 7.208, and $8.810 \mathrm{GHz}$ were confirmed to be obtained by downconversion of these microwaves using the 2D HMA. Each of these first IF components is filtered from each other and downconverted again for the superheterodyne detection. It was confirmed that both the amplitudes and the phases of the detected signals reflect the fluctuations in LHD plasmas. (C) 2010 American Institute of Physics. [doi:10.1063/1.3491197]

\section{INTRODUCTION}

Microwave reflectometry diagnostics has been intensively developed and widely applied to the measurement of electron density profiles and density fluctuations in magnetically confined plasmas. ${ }^{1}$ Rapid progress in microwave technologies has driven the development of various types of reflectometer systems. Microwave imaging reflectometry (MIR) is a multireceiver reflectometry diagnostics, which aims to reconstruct two- or three-dimensional (2D/3D) structures of density fluctuations in turbulent plasmas. ${ }^{2,3}$ In a MIR system, the reflected microwave from a disturbed cutoff surface is focused onto the $2 \mathrm{D}$ receiver antenna array via imaging optics. ${ }^{4,5}$ The $2 \mathrm{D}$ structure of the cutoff surface, which usually corresponds roughly to a magnetic surface, can be obtained with a single-frequency MIR system. The reconstruction of a 3D structure can be realized by using different frequencies that correspond to the different cutoff surfacesthe magnetic surfaces at different radial positions.

The 2D observation of the scattered microwaves in the Large Helical Device (LHD) can be realized with a 2D hornantenna mixer array (2D HMA) ${ }^{6,7}$ It is composed easily by stacking the one-dimensional HMAs, which consist of arrayed quasioptical antenna mixers on a printed-circuit-board enclosed with pyramidal horn apertures. The 2D HMA downconverts the reflected microwaves from the plasmas into the first intermediate frequency (IF) signals by mixing

\footnotetext{
a) Contributed paper, published as part of the Proceedings of the 18th Topical Conference on High-Temperature Plasma Diagnostics, Wildwood, New Jersey, May 2010.

${ }^{b)}$ Electronic mail: yoshinaga.tomokazu@lhd.nifs.ac.jp.
}

those waves with the signal from the first local oscillator (LO) inside each channel's aperture. A downconversion scheme with mixer diodes mounted on microstrip antenna is similar to the previous works on MIR or electron-cyclotron emission imaging systems. ${ }^{2,8,9}$ The advantages of utilizing the HMA are the ease of composing the 2D receiver array and the high gain (directionality) of its horn apertures. However, the imaging optics system becomes rather complicated since the reflected signal waves and the first LO must be simultaneously focused and projected onto the front of the horn aperture ${ }^{10}$ Using a downconversion method for the first IF signals at the receiver antennas significantly simplifies the succeeding signal handling processes, such as transmission, amplification, filtering, and further downconversion. All of these processes use the low cost, mass-produced telecommunications elements that are crucial for a MIR system with many receiver channels.

In the present MIR system in LHD, microwaves at four different frequencies are projected simultaneously onto the target plasmas for the observation of four different cutoff surfaces. In combination with the $2 \mathrm{D}$ HMA having $5 \times 7$ channels, the $3 \mathrm{D}$ observation at radially 4 , toroidally 5 , and poloidally 7 positions can be carried out. The amplitudes of the received waves are detected as well as the phases since many studies have shown both theoretically ${ }^{11-15}$ and experimentally ${ }^{16,17}$ that the amplitudes of the reflected microwaves contain significant information on the fluctuations of the cutoff surfaces. The complex amplitude signals obtained from different positions can be used to deduce the 3D propagation structures in various fluctuations and instabilities via correlational analysis. ${ }^{18}$ As in other reflectometry or interfer- 


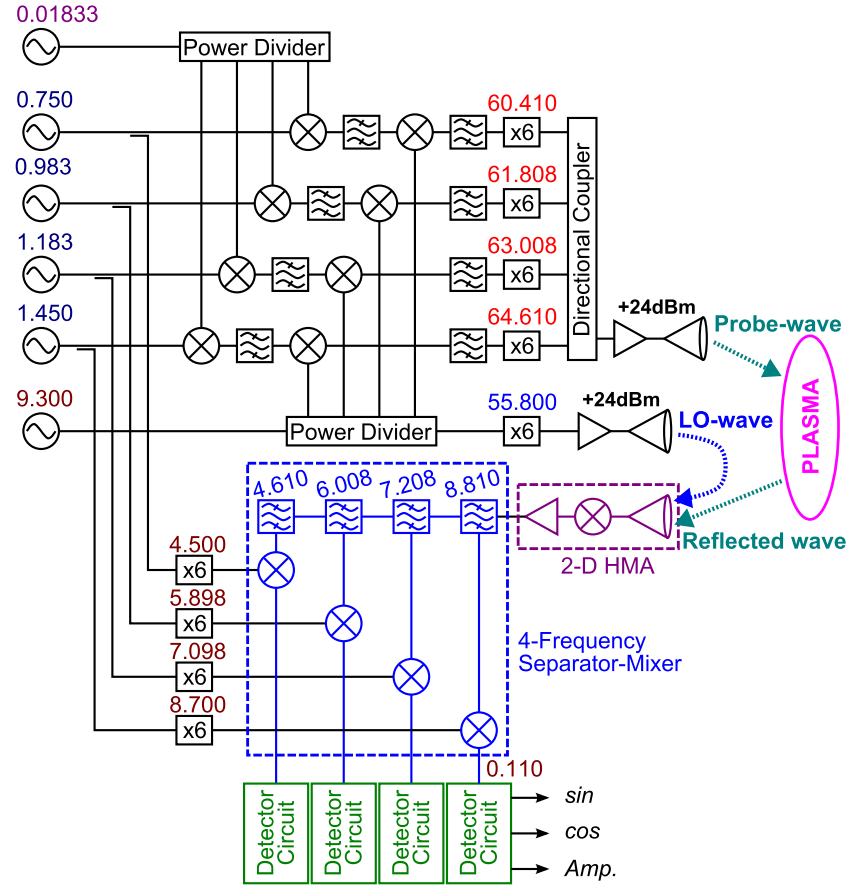

FIG. 1. (Color online) Schematic diagram of the MIR diagnostics system.

ometry systems, the coherency of the illuminating and LO waves is indispensable in detecting the phase of the reflected wave. In addition, oscillator stability is important since the relative phases between different channels must be kept constant, at least during the timescale of the correlational analysis (e.g., Fourier transformation).

This paper describes a four-frequency projection and detection system in Sec. II, the bench testing result is described in Sec. III, an initial result obtained in LHD experiment is introduced and discussed in Sec. IV, and the results are summarized in Sec. V.

\section{MIR DIAGNOSTICS SCHEME IN LHD}

Figure 1 shows a schematic diagram of the MIR diagnostics system in LHD. The four frequencies at 750, 983, 1183 , and $1450 \mathrm{MHz}$ from the frequency-stable crystal oscillators are separately upconverted with $18.33 \mathrm{MHz}$ to form the first IF frequency components. After eliminating the lower sideband frequencies with narrow-band bandpass filters (BPFs) having bandwidths of $\sim 1 \mathrm{MHz}$, the obtained four frequencies are mixed with the carrier wave-a $9.3 \mathrm{GHz}$ signal from the voltage-controlled oscillator. Only the upper sideband frequency components at 10.068, 10.301, 10.501, and $10.768 \mathrm{GHz}$ are used. Here again, it is important to eliminate the lower sideband frequencies; these are reduced to less than $-40 \mathrm{~dB}$ by the BPFs. The four frequencies are separately sextupled and then combined using directional couplers. The microwave (millimeter wave) signal with four frequency components in the V-band (WR-15) at 60.410, $61.808,63.008$, and $64.610 \mathrm{GHz}$ is finally obtained. It is amplified up to $+24 \mathrm{dBm}$ and projected onto the target plasmas via the optics system as an illuminating probe wave. (a)

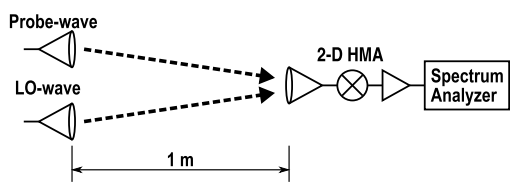

(b)

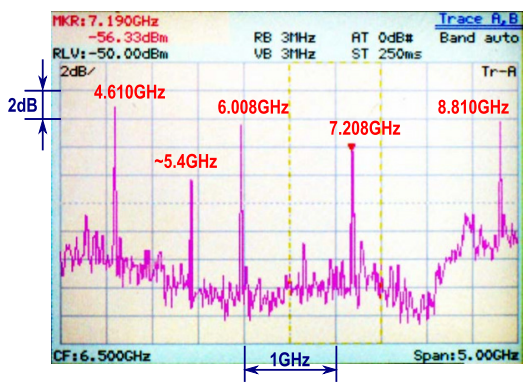

FIG. 2. (Color online) (a) Bench test setup of the four-frequency detection. (b) The first IF spectrum obtained from bench testing in (a).

The reflected waves from the cutoff surfaces in the target plasmas are focused via the same optics onto the aperture plane of 2D HMA. The received wave is mixed with the first LO signal at $55.800 \mathrm{GHz}$ inside each small horn aperture of the HMA. The first LO is generated by dividing and sextupling the carrier frequency of the illuminating wave at $9.300 \mathrm{GHz}$. It is also amplified up to $+24 \mathrm{dBm}$ and projected onto the HMA aperture, focused by the imaging optics system. The downconverted first IF signal obtained from the HMA, which consists of four frequency components at $4.610,6.008,7.208$, and $8.810 \mathrm{GHz}$, is resolved into the signals with their respective frequencies using the microstrip-line BPF circuits. ${ }^{19,20}$

The separated first IF signals are downconverted again by mixing with the respective four second LO signals (4.500, $5.898,7.098$, and $8.700 \mathrm{GHz}$ ). The signals from the same crystal oscillators for the illuminating wave are divided and used for these second LO signals, as the first LO generation. Thus, the second IF signals, all at $110 \mathrm{MHz}$, are finally obtained. Their amplitudes and phases are detected using the power-detector and the quadrature-demodulator circuits. Narrow-band, surface acoustic wave filters with a bandwidth of $\sim 4 \mathrm{MHz}$ are applied to the detection circuits with gallium-arsenide (GaAs) low-noise amplifiers for noise reduction. The electronic components mounted on the detection circuit are those used in advanced mobile communication modules, such as global positioning systems or mobile phones, and are provided at low cost. It is confirmed that the oscillators are stable, at least over a timescale of $1 \mathrm{~min}$. Therefore, the background amplitude and phase signals can be considered constant during the LHD discharges, which are typically several seconds long, and the correlational analysis is applicable.

\section{BENCH TEST OF FOUR-FREQUENCY DETECTION}

The four-frequency generation/detection scheme was verified by a simple bench test. Figure 2(a) shows the experimental setup. The illumination wave with four frequency components and the first $\mathrm{LO}$ wave are projected from the horn antennas pointing at the receiver HMA. Figure 2(b) is a 

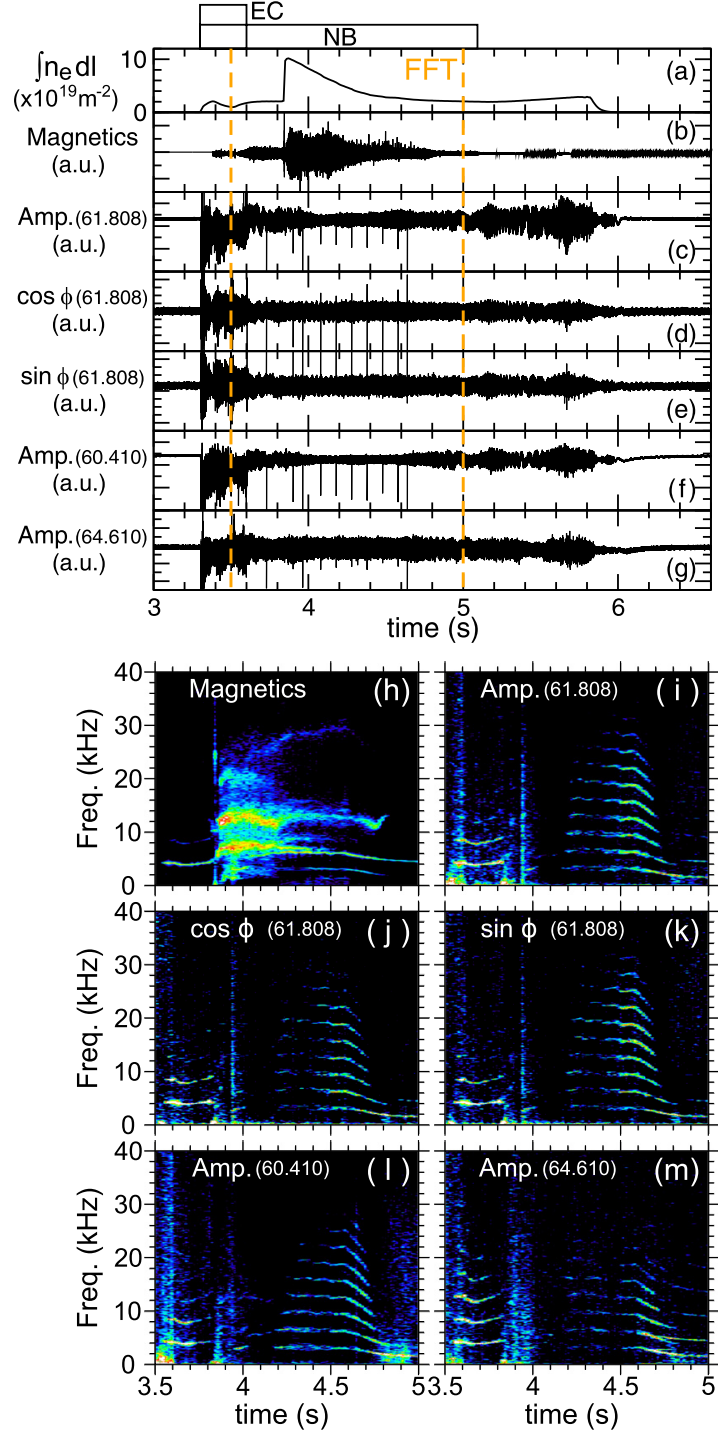

FIG. 3. (Color online) Waveforms of a typical discharge in high iontemperature operation (Shot No. 96253): (a) the line-density, (b) magnetic probe signal, (c) MIR amplitude signal, [(d) and (e)] MIR quadrature-phase signals working at $61.808 \mathrm{GHz},[(\mathrm{f})$ and $(\mathrm{g})]$ MIR amplitude signals working at 60.410 and $64.610 \mathrm{GHz}$, respectively, and [(h)-(m)] the FFT spectra of (b) $-(\mathrm{g})$, respectively.

photograph of the spectrum analyzer (Anritsu MS2665C) that shows the first IF spectra from the HMA. The intended four frequency components are observed, while there is an unexpected frequency component found at $\sim 5.4 \mathrm{GHz}$. This frequency corresponds to the IF frequency between the septupled frequency of $10.068 \mathrm{GHz}$ in the illuminating wave and that of $9.300 \mathrm{GHz}$ for the LO wave. This unnecessary frequency does not disturb the detection of the intended frequencies since it is eliminated in the downconversion process into the second IF signals.

\section{INITIAL RESULT IN LHD AND DISCUSSION}

The four-frequency projection/detection system was installed in LHD for experiments conducted in 2009 together with the redesigned optics system. ${ }^{10}$ Figure 3 compares the MIR signals under a high ion-temperature discharge with the signals from other diagnostics. The signals with the illumi- nating frequencies $\left(f_{\mathrm{IL}}\right)$ at $60.410,61.808$, and $64.610 \mathrm{GHz}$ from the central HMA channel of the MIR, whose field of view is on the optical axis, are plotted. In Figs. 3(c)-3(g), the fluctuations in the MIR signals appear to be excited during the period when there is some finite electron density, as shown in Fig. 3(a). Here, the signals denoted by $\cos \phi$ [Fig. $3(\mathrm{~d})]$ and $\sin \phi$ [Fig. 3(e)] correspond to the quadraturedemodulated signals into the in-phase and the quadraturephase, respectively. It is suggested that the present MIR system can obtain the fluctuation signals from the plasma itself rather than from the electron-cyclotron or the neutral-beam heating pulses. Figures $3(\mathrm{i})-3(\mathrm{~m})$ show the fast Fouriertransform (FFT) spectra of the MIR signals. The fluctuations with a fundamental frequency of several kilohertz, which are found at $t \sim 3.5-3.8 \mathrm{~s}$ and $t \sim 4.4-4.8 \mathrm{~s}$ in the MIR spectra, can also be found in the magnetic signal's spectra shown in Fig. 3(h). This also supports the contention that the MIR system is observing fluctuations in the plasmas.

Meanwhile, some differences exist between the spectra from the two diagnostics systems. Some frequency components appear only in the magnetic signal (e.g., $f \sim 7,14$, and $28 \mathrm{kHz}$ at $t=4.2-4.8 \mathrm{~s}$ ). This would originate from inherent differences in their observation areas. The MIR is a local diagnostics in the vicinity of the cutoff surface, while the magnetic diagnostics reflects fluctuations in a wide area of the plasma. Therefore, the signals that appear only in the magnetic signal would be those arising in the inner region of the cutoff surfaces observed on the MIR.

At $t=4.2 \mathrm{~s}$, the cutoff surfaces of three $f_{\text {IL }}$ are all located outside the last closed magnetic flux surface (LCFS) ( $\rho$ $>1$ ), where $\rho$ is the normalized minor radius. They are placed within $\sim 5 \mathrm{~cm}$ of each other and move inward with the density decrease, as shown in Fig. 3(a), and reach the edge plasma region inside the LCFS $(\rho \sim 0.9-1)$ at $t$ $=4.6 \mathrm{~s}$, roughly maintaining their distances. Although the FFT spectra of the MIR amplitudes are all similar, the timings that the fluctuation signals accompanying the high-order harmonics appear clearly are slightly different, as shown in Figs. 3(i), 3(1), and 3(m). Those harmonic oscillations appear first $(t \sim 4.2 \mathrm{~s})$ in the signal with $f_{\mathrm{IL}}=60.410 \mathrm{GHz}$ [Fig. 3(1)], become clear in the signal with $f_{\mathrm{IL}}=61.808 \mathrm{GHz}$ at $t$ $\sim 4.4 \mathrm{~s}$ [Fig. 3(i)], and finally appear in the signal with $f_{\mathrm{IL}}$ $=64.610 \mathrm{GHz}$ after $t \sim 4.6 \mathrm{~s}$ [Fig. $3(\mathrm{~m})]$. In speculation, some oscillations around the LCFS might be observed when the observation area of MIR moves inward across the LCFS with the decrease in the electron density.

The high-order harmonic frequency observed in the MIR signals can arise for many reasons. First, the density fluctuations in the edge region might not act in a sinusoidal manner. On the other hand, when the fluctuation level is very large, or the structures (wavelengths) are quite small, the signals of the reflected wave would be strongly distorted. ${ }^{5,11,15} \mathrm{Al}$ though the physical phenomena that caused the high-order harmonic signals of the MIR is beyond the scope of this paper, it is an important issue to address in future work on the observation of the fluctuation structure. 


\section{SUMMARY}

A simultaneous projection and detection system of four different frequencies for MIR diagnostics was developed for the $3 \mathrm{D}$ observation of the electron density fluctuations in LHD plasmas. The microwaves at 60.410, 61.808, 63.008, and $64.610 \mathrm{GHz}$ are projected simultaneously onto the target plasmas. The reflected waves from the plasmas are mixed with the LO wave at $55.800 \mathrm{GHz}$ to be downconverted into four IF components by the 2D HMA. A simple bench test confirmed that the first IF signals at 4.610, 6.008, 7.208, and $8.810 \mathrm{GHz}$ were obtained as intended. The system was applied to LHD for the 13th experimental campaign in 2009 and started operation. Fluctuations were observed for both the amplitude and the phase signals during plasma discharges.

\section{ACKNOWLEDGMENTS}

This work was supported by the National Institute of Natural Sciences Imaging Science Project (Grant No. NIFS09KEIN0021), by the National Institute of Fusion Science (Grant No. NIFS09ULPP525), and by the Grant-in-Aid for Scientific Research (Grant No. 21246140). The authors thank S. Sugito and T. Nishimura from the Department of Engineering and Technical Services at NIFS for their technical supports.

${ }^{1}$ C. Laviron, A. J. H. Donné, M. E. Manso, and J. Sanchez, Plasma Phys. Controlled Fusion 38, 905 (1996).

${ }^{2}$ H. Park, C. C. Chang, B. H. Deng, C. W. Domier, A. J. H. Donné, K. Kawahata, C. Liang, X. P. Liang, H. J. Lu, N. C. Luhmann, A. Mase, H. Matsuura, E. Mazzucato, A. Miura, K. Mizuno, T. Munsat, Y. Nagayama, M. J. van de Pol, J. Wang, Z. G. Xia, and W.-K. Zhang, Rev. Sci. Instrum. 74, 4239 (2003).
${ }^{3}$ H. Park, E. Mazzucato, T. Munsat, C. W. Domier, M. Johnson, N. C. Luhmann, J. Wang, Z. Xia, I. G. J. Classen, A. J. H. Donné, and M. J. van de Pol, Rev. Sci. Instrum. 75, 3787 (2004).

${ }^{4}$ E. Mazzucato, Nucl. Fusion 41, 203 (2001).

${ }^{5}$ E. Mazzucato, T. Munsat, H. Park, B. H. Deng, C. W. Domier, N. C. Luhmann, A. J. H. Donné, and M. J. van de Pol, Phys. Plasmas 9, 1955 (2002).

${ }^{6}$ D. Kuwahara, S. Tsuji-Iio, Y. Nagayama, T. Yoshinaga, M. Sugito, Z. Shi, S. Yamaguchi, Y. Kogi, and A. Mase, J. Plasma Fusion Res. 8, 649 (2009).

${ }^{7}$ D. Kuwahara, S. Tsuji-Iio, Y. Nagayama, T. Yoshinaga, Z. Shi, S. Yamaguchi, M. Sugito, Y. Kogi, and A. Mase, J. Plasma Fusion Res. 9, 125 (2010).

${ }^{8}$ P. L. Hsu, B. H. Deng, J. Wang, C. W. Domier, and N. C. Luhmann, Rev. Sci. Instrum. 72, 364 (2001).

${ }^{9}$ T. Munsat, E. Mazzucato, H. Park, B. H. Deng, C. W. Domier, N. C. Luhmann, J. Wang, Z. G. Xia, A. J. H. Donné, and M. van de Pol, Rev. Sci. Instrum. 74, 1426 (2003).

${ }^{10}$ T. Yoshinaga, Y. Nagayama, D. Kuwahara, H. Tsuchiya, S. Yamaguchi, Y. Kogi, S. Tsuji-Iio, H. Hojo, and A. Mase, J. Plasma Fusion Res. 5, 30 (2010).

${ }^{11}$ J. H. Irby, S. Horne, I. H. Hutchinson, and P. C. Stek, Plasma Phys. Controlled Fusion 35, 601 (1993).

${ }^{12}$ E. Mazzucato and R. Nazikian, Rev. Sci. Instrum. 66, 2237 (1995).

${ }^{13}$ E. Mazzucato, Rev. Sci. Instrum. 69, 1691 (1998).

${ }^{14}$ E. Mazzucato, Plasma Phys. Controlled Fusion 46, 1271 (2004).

${ }^{15}$ A. Ejiri, T. Yamada, Y. Adachi, O. Watanabe, and Y. Takase, Plasma Phys. Controlled Fusion 50, 065003 (2008).

${ }^{16}$ T. L. Rhodes, W. A. Peebles, E. J. Doyle, P. Pribyl, M. Gilmore, R. A. Moyer, and R. D. Lehmer, Plasma Phys. Controlled Fusion 40, 493 (1998).

${ }^{17}$ G. D. Conway, G. Vayakis, J. A. Fessey, and D. V. Bartlett, Rev. Sci. Instrum. 70, 3921 (1999).

${ }^{18}$ Z. Shi, Y. Nagayama, S. Yamaguchi, D. Kuwahara, T. Yoshinaga, S. Sugito, Y. Hirano, H. Koguchi, S. Kiyama, H. Sakakita, K. Yambe, and C. Michael, J. Plasma Fusion Res. 5, S1019 (2010).

${ }^{19}$ Y. Kogi, T. Sakoda, A. Mase, N. Ito, Y. Yokota, S. Yamaguchi, Y. Nagayama, S. H. Jeong, M. Kwon, and K. Kawahata, Rev. Sci. Instrum. 79, 10F115 (2008).

${ }^{20}$ S. Yamaguchi, Y. Nagayama, D. Kuwahara, T. Yoshinaga, Z. B. Shi, Y. Kogi, and A. Mase, Rev. Sci. Instrum. 79, 10F111 (2008). 